\title{
Dirac operator coupled to bosons
}

\author{
A. A. Boitsev ${ }^{1}$, H. Neidhardt ${ }^{2}$, I. Y. Popov ${ }^{1}$ \\ ${ }^{1}$ ITMO University, Kronverkskiy, 49, St. Petersburg, 197101, Russia \\ ${ }^{2}$ Weierstrass Institute for Applied Analysis and Stochastics, \\ Mohrenststr. 39, D-10117, Berlin, Germany
}

boitsevanton@gmail.com, hagen.neidhardt@wias-berlin.de,popov1955@gmail.com

PACS 02.30.Tb

DOI 10.17586/2220-8054-2016-7-2-332-339

\begin{abstract}
We consider a model of point-like interaction between electrons and bosons in a cavity. The electrons are relativistic and are described by a Dirac operator on a bounded interval while the bosons are treated by second quantization. The model fits into the extension theory of symmetric operators. Our main technical tool to handle the model is the so-called boundary triplet approach to extensions of symmetric operators. The approach allows explicit computation of the Weyl function.
\end{abstract}

Keywords: Dirac operator, bosons, operator extension, boundary triplet, second quantization.

Received: 13 January 2016

\section{Introduction}

The problem of electro-magnetic field's influence on an electron's spectral and transport properties has attracted great interest from physicists due to its importance both from fundamental and engineering viewpoints (see, e.g., [1] and references therein). It is important to find solvable models to describe this phenomenon. We present a simple model in the framework of extension theory of symmetric operators. The electro-magnetic field can be described classically or it can be quantized. Using a classical description, such a model was proposed in in [2]. The case of quantized electro-magnetic field is more complicated. As an example of this type of model, we can mention [3], [4]. In the present paper, we suggest a model of point-like interaction between a relativistic fermion (the Dirac operator) and bosons (infinite matrix operator in the Fock space). We use the boundary triplet approach to describe extensions of symmetric operators (see, e.g., $[8-13,15])$.

In the following, we consider a particular example of point interaction for a quantum system $\left\{\mathfrak{D}, D_{0}\right\}$ with a quantum reservoir $\{\mathfrak{T}, T\}$, where $D_{0}$ denotes the self-adjoint Dirac operator defined in the Hilbert space $\mathfrak{D}=L^{2}\left(\Delta, \mathbb{C}^{2}\right)$, where $\Delta=(a ; b)$, and $T$ is the so-called boson operator defined in the Hilbert space $\mathfrak{T}=l_{2}\left(\mathbb{N}_{0}\right)$. Before introducing the operators, let us slightly specify the approach.

At first, we consider a system consisting of a quantum system $\left\{\mathfrak{D}, D_{0}\right\}$ and a quantum reservoir $\{\mathfrak{T}, T\}$. This system's composed Hamiltonian is given by the self-adjoint operator:

$$
L_{0}:=D_{0} \otimes I_{\mathfrak{T}}+I_{\mathfrak{D}} \otimes T,
$$

which acts in $\mathfrak{L}:=\mathfrak{D} \otimes \mathfrak{T}$. In order to describe the interaction, we restrict the operator $D_{0}$ to a densely defined closed symmetric operator $D$ and consider the densely defined symmetric operator:

$$
L:=D \otimes I_{\mathfrak{T}}+I_{\mathfrak{D}} \otimes T
$$


with $\operatorname{dom}(L)=\operatorname{dom}(D) \otimes \operatorname{dom}(T)$, where:

$$
\begin{gathered}
(D f)(x):=-i c \frac{d}{d x} \otimes \sigma_{1} f(x)+\frac{c^{2}}{2} \otimes \sigma_{3} f(x), \quad x \in \Delta, \\
f \in \operatorname{dom}(D):=W_{0}^{1,2}\left(\Delta, \mathbb{C}^{2}\right):=\left\{f \in W^{1,2}\left(\Delta, \mathbb{C}^{2}\right): f(a)=f(b)=0\right\} .
\end{gathered}
$$

Here,

$$
\sigma_{1}:=\left(\begin{array}{cc}
0 & 1 \\
1 & 0
\end{array}\right) \quad \text { and } \quad \sigma_{3}:=\left(\begin{array}{cc}
1 & 0 \\
0 & -1
\end{array}\right)
$$

$T$ denotes a boson operator in the Hilbert space $\mathfrak{T}=l_{2}\left(\mathbb{N}_{0}\right)$ such that:

$$
\begin{aligned}
T \vec{\xi} & =T\left\{\xi_{k}\right\}_{k \in \mathbb{N}_{0}}=\left\{k \xi_{k}\right\}_{k \in \mathbb{N}_{0}}, \\
\vec{\xi} & =\left\{\xi_{k}\right\}_{k \in \mathbb{N}_{0}} \in \operatorname{dom}(T):=\left\{\left\{\xi_{k}\right\}_{k \in \mathbb{N}_{0}} \in l_{2}\left(\mathbb{N}_{0}\right):\left\{k \xi_{k}\right\}_{k \in \mathbb{N}_{0}} \in l_{2}\left(\mathbb{N}_{0}\right)\right\} .
\end{aligned}
$$

To construct self-adjoint extensions we use the boundary triplet approach. Notice that there are extensions which do not correspond to any interaction between both subsystems. From the physical point of view it is very important to obtain those extensions which realistically describe point interactions.

\section{Preliminaries}

\subsection{Linear relations}

A linear relation $\Theta$ in $\mathcal{H}$ is a closed linear subspace of $\mathcal{H} \oplus \mathcal{H}$. The set of all linear relations in $\mathcal{H}$ is denoted by $\widetilde{\mathcal{C}}(\mathcal{H})$. We also denote by $\mathcal{C}(\mathcal{H})$ the set of all closed linear (not necessarily densely defined) operators in $\mathcal{H}$. Identifying each operator $T \in \mathcal{C}(\mathcal{H})$ with its graph $\operatorname{gr}(T)$, we regard $\mathcal{C}(\mathcal{H})$ as a subset of $\widetilde{\mathcal{C}}(\mathcal{H})$.

The role of the set $\widetilde{\mathcal{C}}(\mathcal{H})$ in extension theory becomes apparent from Proposition 2.3. However, it's role in the operator theory is substantially motivated by the following circumstances: in contrast to $\mathcal{C}(\mathcal{H})$, the set $\widetilde{\mathcal{C}}(\mathcal{H})$ is closed with respect to taking inverse and adjoint relations $\Theta^{-1}$ and $\Theta^{*}$, respectively. The latter is given by: $\Theta^{-1}=\{\{g, f\}:\{f, g\} \in \Theta\}$ and

$$
\Theta^{*}=\left\{\left(\begin{array}{c}
k \\
k^{\prime}
\end{array}\right):\left(h^{\prime}, k\right)=\left(h, k^{\prime}\right) \text { for all }\left(\begin{array}{c}
h \\
h^{\prime}
\end{array}\right) \in \Theta\right\} .
$$

A linear relation $\Theta$ is called symmetric if $\Theta \subset \Theta^{*}$ and self-adjoint if $\Theta=\Theta^{*}$.

\subsection{Boundary triplets and proper extensions}

Let us briefly recall some basic facts regarding boundary triplets. Let $A$ be a densely defined closed symmetric operator with equal deficiency indices $n_{ \pm}(A):=\operatorname{dim}\left(\mathfrak{N}_{ \pm \mathrm{i}}\right), \mathfrak{N}_{z}:=$ $\operatorname{ker}\left(A^{*}-z\right), z \in \mathbb{C}_{ \pm}$, acting on some separable Hilbert space $\mathfrak{H}$.

\section{Definition 2.1.}

(i) $A$ closed extension $\widetilde{A}$ of $A$ is called proper if $\operatorname{dom}(A) \subset \operatorname{dom}(\widetilde{A}) \subset \operatorname{dom}\left(A^{*}\right)$.

(ii) Two proper extensions $\widetilde{A}^{\prime}, \widetilde{A}$ are called disjoint if $\operatorname{dom}\left(\widetilde{A}^{\prime}\right) \cap \operatorname{dom}(\widetilde{A})=\operatorname{dom}(A)$ and transversal if in addition $\operatorname{dom}\left(\widetilde{A}^{\prime}\right)+\operatorname{dom}(\widetilde{A})=\operatorname{dom}\left(A^{*}\right)$.

We denote by $\operatorname{Ext}_{\mathrm{A}}$ the set of all proper extensions of $A$ completed by the non-proper extensions $A$ and $A^{*}$. For instance, any self-adjoint or maximally dissipative (accumulative) extension is proper. 
Definition 2.2 ( [11]). A triplet $\Pi=\left\{\mathcal{H}, \Gamma_{0}, \Gamma_{1}\right\}$, where $\mathcal{H}$ is an auxiliary Hilbert space and $\Gamma_{0}, \Gamma_{1}: \operatorname{dom}\left(A^{*}\right) \rightarrow \mathcal{H}$ are linear mappings, is called a boundary triplet for $A^{*}$ if the "abstract Green's identity",

$$
\left(A^{*} f, g\right)-\left(f, A^{*} g\right)=\left(\Gamma_{1} f, \Gamma_{0} g\right)-\left(\Gamma_{0} f, \Gamma_{1} g\right), \quad f, g \in \operatorname{dom}\left(S^{*}\right),
$$

is satisfied and the mapping $\Gamma:=\left(\Gamma_{0}, \Gamma_{1}\right)^{\top}: \operatorname{dom}\left(A^{*}\right) \rightarrow \mathcal{H} \oplus \mathcal{H}$ is surjective, i.e. $\operatorname{ran}(\Gamma)=$ $\mathcal{H} \oplus \mathcal{H}$.

A boundary triplet $\Pi=\left\{\mathcal{H}, \Gamma_{0}, \Gamma_{1}\right\}$ for $A^{*}$ always exists whenever $n_{+}(A)=n_{-}(A)$. Note also that $n_{ \pm}(A)=\operatorname{dim}(\mathcal{H})$ and $\operatorname{ker}\left(\Gamma_{0}\right) \cap \operatorname{ker}\left(\Gamma_{1}\right)=\operatorname{dom}(A)$.

With any boundary triplet $\Pi$ one associates two canonical self-adjoint extensions $A_{j}:=$ $S^{*} \uparrow \operatorname{ker}\left(\Gamma_{j}\right), j \in\{0,1\}$. Conversely, for any self-adjoint extension $A_{0}=S_{0}^{*} \in \operatorname{Ext}_{\mathrm{S}}$ there exists a (non-unique) boundary triplet $\Pi=\left\{\mathcal{H}, \Gamma_{0}, \Gamma_{1}\right\}$ for $A^{*}$ such that $A_{0}:=A^{*} \uparrow \operatorname{ker}\left(\Gamma_{0}\right)$.

Using the concept of boundary triplets, one can parametrize all proper extensions of $A$ in the following way.

Proposition 2.3 ( $[9,13])$. Let $\Pi=\left\{\mathcal{H}, \Gamma_{0}, \Gamma_{1}\right\}$ be a boundary triplet for $A^{*}$. Then the mapping:

$$
\operatorname{Ext}_{\mathrm{A}} \ni \widetilde{\mathrm{A}} \rightarrow \Gamma \operatorname{dom}(\widetilde{\mathrm{A}})=\left\{\left(\Gamma_{0} \mathrm{f}, \Gamma_{1} \mathrm{f}\right)^{\top}: \mathrm{f} \in \operatorname{dom}(\widetilde{\mathrm{A}})\right\}=: \Theta \in \widetilde{\mathcal{C}}(\mathcal{H})
$$

establishes a bijective correspondence between the sets $\operatorname{Ext}_{\mathrm{A}}$ and $\widetilde{\mathcal{C}}(\mathcal{H})$. We write $\widetilde{A}=A_{\Theta}$ if $\widetilde{A}$ corresponds to $\Theta$ by (2.2). Moreover, the following holds:

(i) $A_{\Theta}^{*}=A_{\Theta^{*}}$, in particular, $A_{\Theta}^{*}=A_{\Theta}$ if and only if $\Theta^{*}=\Theta$.

(ii) $A_{\Theta}$ is symmetric (self-adjoint) if and only if $\Theta$ is symmetric (self-adjoint).

(iii) The extensions $A_{\Theta}$ and $A_{0}$ are disjoint (transversal) if and only if there is a closed (bounded) operator $B$ such that $\Theta=\operatorname{gr}(B)$. In this case (2.2) takes the form:

$$
A_{\Theta}:=A_{\operatorname{gr}(B)}=A^{*} \uparrow \operatorname{ker}\left(\Gamma_{1}-B \Gamma_{0}\right) .
$$

In particular, $A_{j}:=A^{*} \uparrow \operatorname{ker}\left(\Gamma_{j}\right)=A_{\Theta_{j}}, j \in\{0,1\}$, where $\Theta_{0}:=\left(\begin{array}{c}\{0\} \\ \mathcal{H}\end{array}\right)$ and $\Theta_{1}:=\left(\begin{array}{c}\mathcal{H} \\ \{0\}\end{array}\right)=\operatorname{gr}(\mathbb{O})$ where $\mathbb{O}$ denotes the zero operator in $\mathcal{H}$. Note also that $\widetilde{\mathcal{C}}(\mathcal{H})$ contains the trivial linear relations $\{0\} \times\{0\}$ and $\mathcal{H} \times \mathcal{H}$ corresponding to $A$ and $A^{*}$, respectively, for any boundary triplet $\Pi$.

\subsection{Gamma field and Weyl function}

It is well known that the Weyl function is an important tool in the direct and inverse spectral theory of Sturm-Liouville operators. In $[8,9]$, the concept of Weyl function was generalized to the case of an arbitrary symmetric operator $A$ with $n_{+}(A)=n_{-}(A) \leq \infty$. Following [9] we briefly recall basic facts on Weyl functions and Gamma fields associated with a boundary triplet $\Pi$.

Definition 2.4 ( $[8,9])$. Let $\Pi=\left\{\mathcal{H}, \Gamma_{0}, \Gamma_{1}\right\}$ be a boundary triplet for $A^{*}$ and $A_{0}=A^{*} \uparrow$ $\operatorname{ker}\left(\Gamma_{0}\right)$. The operator valued functions $\gamma(\cdot): \rho\left(A_{0}\right) \rightarrow[\mathcal{H}, \mathcal{H}]$ and $M(\cdot): \rho\left(A_{0}\right) \rightarrow[\mathcal{H}]$ defined by the following:

$$
\gamma(z):=\left(\Gamma_{0} \uparrow \mathfrak{N}_{z}\right)^{-1}, \quad \mathfrak{N}_{z}=\operatorname{ker}\left(A^{*}-z\right) \quad \text { and } \quad M(z):=\Gamma_{1} \gamma(z), \quad z \in \rho\left(A_{0}\right),
$$

are called the Gamma field and the Weyl function, respectively, corresponding to the boundary triplet $\Pi$. 
Clearly, the Weyl function can equivalently be defined by:

$$
M(z) \Gamma_{0} f_{z}=\Gamma_{1} f_{z}, \quad f_{z} \in \mathfrak{N}_{z}, \quad z \in \rho\left(A_{0}\right) .
$$

The Gamma field $\gamma(\cdot)$ and the Weyl function $M(\cdot)$ in (2.4) are well defined. Moreover, both $\gamma(\cdot)$ and $M(\cdot)$ are holomorphic on $\rho\left(A_{0}\right)$ and the following relations:

$$
\gamma(z)=\left(I+(z-\zeta)\left(A_{0}-z\right)^{-1}\right) \gamma(\zeta), \quad z, \zeta \in \rho\left(A_{0}\right),
$$

and

$$
M(z)-M(\zeta)^{*}=(z-\bar{\zeta}) \gamma(\zeta)^{*} \gamma(z), \quad z, \zeta \in \rho\left(A_{0}\right)
$$

hold. Identity (2.6) yields that $M(\cdot)$ is $[\mathcal{H}]$-valued Nevanlinna function $(M(\cdot) \in R[\mathcal{H}])$, i.e. $M(\cdot)$ is $[\mathcal{H}]$-valued holomorphic function on $\mathbb{C}_{ \pm}$satisfying:

$$
M(z)=M(\bar{z})^{*} \quad \text { and } \quad \frac{\operatorname{Im}(M(z))}{\operatorname{Im}(z)} \geq 0, \quad z \in \mathbb{C}_{+} \cup \mathbb{C}_{-} .
$$

It also follows also from (2.6) that $0 \in \rho(\operatorname{Im}(M(z)))$ for all $z \in \mathbb{C}_{ \pm}$.

A Weyl function $M(\cdot)$ is said to be of a scalar type if there exists a scalar Nevanlinna function $m(\cdot)$ such that the the representation:

$$
M(z)=m(z) I_{\mathcal{H}}, \quad z \in \mathbb{C}_{+},
$$

holds where $I_{\mathcal{H}}$ is the identity operator in $\mathcal{H}$, see [5]. Obviously, $M(\cdot)$ is of a scalar type if $n_{ \pm}(A)=1$.

\subsection{Krein-type formula for resolvents}

Let $\Pi=\left\{\mathcal{H}, \Gamma_{0}, \Gamma_{1}\right\}$ be a boundary triplet for $A^{*}, M(\cdot)$ and $\gamma(\cdot)$ the corresponding Weyl function and Gamma field, respectively. For any proper (not necessarily self-adjoint) extension $\widetilde{A}_{\Theta} \in \operatorname{Ext}_{\mathrm{A}}$ with non-empty resolvent set $\rho\left(\widetilde{A}_{\Theta}\right)$, the following Krein-type formula holds (cf. [8-10]):

$$
\left(A_{\Theta}-z\right)^{-1}-\left(A_{0}-z\right)^{-1}=\gamma(z)(\Theta-M(z))^{-1} \gamma^{*}(\bar{z}), \quad z \in \rho\left(A_{0}\right) \cap \rho\left(A_{\Theta}\right) .
$$

Formula (2.8) extends the known Krein formula for canonical resolvents to the case of any $A_{\Theta} \in \operatorname{Ext}_{\mathrm{A}}$ with $\rho\left(S_{\Theta}\right) \neq \emptyset$. Moreover, due to relations (2.2), (2.3) and (2.4) formula (2.8) is connected with the boundary triplet $\Pi$. We emphasize that this connection makes it possible to apply the Krein-type formula (2.8) to boundary value problems.

\subsection{Direct sum of operators}

Let $S_{n}$ be a densely defined closed symmetric operator in a Hilbert space $\mathfrak{H}_{n}$ with $n_{+}\left(S_{n}\right)=n_{-}\left(S_{n}\right) \leq \infty, n \in \mathbb{N}$. Consider the operator $S:=\bigoplus_{n=1}^{\infty} S_{n}$ acting in $\mathfrak{H}:=\bigoplus_{n=1}^{\infty} \mathfrak{H}_{n}$, the Hilbert direct sum of Hilbert spaces $\mathfrak{H}_{n}$. By definition, $\stackrel{n=1}{\mathfrak{H}}=\left\{f=\oplus_{n=1}^{\infty} f_{n}: \quad{ }_{n=1} \in\right.$ $\left.\mathfrak{H}_{n}, \quad \sum_{n=1}^{\infty}\left\|f_{n}\right\|^{2}<\infty\right\}$. From this, it is apparent that:

$$
S^{*}=\bigoplus_{n=1}^{\infty} S_{n}^{*}, \quad \operatorname{dom}\left(S^{*}\right)=\left\{f=\oplus_{n=1}^{\infty} f_{n} \in \mathfrak{H}: \quad f_{n} \in \operatorname{dom}\left(S_{n}^{*}\right), \quad \sum_{n=1}^{\infty}\left\|S_{n}^{*} f_{n}\right\|^{2}<\infty\right\}
$$


Theorem 2.5 (Theorem 2.10 of [7]). Let $\Pi_{n}=\left\{\mathcal{H}_{n}, \Gamma_{0}^{n}, \Gamma_{1}^{n}\right\}$ be a boundary triplet for $S_{n}^{*}$ and $M_{n}(\cdot)$ the corresponding Weyl function, $n \in \mathbb{N}$. A direct sum $\Pi=\oplus_{n=1}^{\infty} \Pi_{n}$ forms an ordinary boundary triplet for the operator $S^{*}=\bigoplus_{n=1}^{\infty} S_{n}^{*}$ if and only if

$$
\sup _{n}\left\|M_{n}(i)\right\|_{\mathcal{H}_{n}}<\infty, \quad \sup _{n}\left\|\left(\operatorname{Im}\left(M_{n}(i)\right)\right)^{-1}\right\|_{\mathcal{H}_{n}}
$$

\section{Weyl function computation}

Let us describe the procedure to obtain all extension of the operator (1.1). Firstly, let us consider symmetric Dirac operator, defined by (1.2). The adjoint operator $D^{*}$ appears as:

$$
\begin{aligned}
\left(D^{*} f\right)(x) & =-i c \frac{d}{d x} \otimes \sigma_{1} f(x)+\frac{c^{2}}{2} \otimes \sigma_{3} f(x), \quad x \in \Delta, \\
f \in \operatorname{dom}\left(D^{*}\right) & =W^{1,2}\left(\Delta, \mathbb{C}^{2}\right) .
\end{aligned}
$$

The triplet $\Pi_{D}=\left\{\mathcal{H}^{D}, \Gamma_{0}^{D}, \Gamma_{1}^{D}\right\}, \mathcal{H}^{D}:=\mathbb{C}^{2}$,

$$
\begin{aligned}
& \Gamma_{0}^{D}\left(\begin{array}{l}
f_{1} \\
f_{2}
\end{array}\right):=\frac{1}{\sqrt{2}}\left(\begin{array}{l}
f_{1}(a)+f_{1}(b) \\
f_{1}(a)-f_{1}(b)
\end{array}\right), \\
& \Gamma_{1}^{D}\left(\begin{array}{l}
f_{1} \\
f_{2}
\end{array}\right):=\frac{i c}{\sqrt{2}}\left(\begin{array}{l}
f_{2}(a)-f_{2}(b) \\
f_{2}(a)+f_{2}(b)
\end{array}\right),
\end{aligned}
$$

$f \in \operatorname{dom}\left(D^{*}\right)$, forms a boundary triplet for $D^{*}$. The Gamma field and the Weyl function are given by:

$$
\gamma^{D}(z)\left(\begin{array}{l}
\xi_{1} \\
\xi_{2}
\end{array}\right)=\frac{1}{\sqrt{2}}\left(\begin{array}{cc}
\frac{\cos (k(z)(x-\nu))}{\cos (k(z) d)} & \frac{\sin (k(z)(x-\nu))}{\sin (k(z) d)} \\
i k_{1}(z) \frac{\sin (k(z)(x-\nu))}{\cos (k(z) d} & i k_{1}(z) \frac{\cos (k(z)(x-\nu))}{\sin (k(z) d)}
\end{array}\right)\left(\begin{array}{l}
\xi_{1} \\
\xi_{2}
\end{array}\right)
$$

$z \in \mathbb{C}_{ \pm}$. Here,

$$
k(z):=\frac{1}{c} \sqrt{z^{2}-\frac{c^{4}}{4}}, \quad z \in \mathbb{C}
$$

where the branch of the multifunction $k(\cdot)$ is fixed by the condition $k(x)>0$ for $x>\frac{c^{2}}{2}$. Notice that $k(\cdot)$ is holomorphic in $\mathbb{C} \backslash\left[-\frac{c^{2}}{2}, \frac{c^{2}}{2}\right]$. Furthermore,

$$
k_{1}(z):=\frac{c k(z)}{z+\frac{c^{2}}{2}}, \quad z \in \mathbb{C} .
$$

which is also holomorphic in $\mathbb{C} \backslash\left[-\frac{c^{2}}{2}, \frac{c^{2}}{2}\right]$. The function $k_{1}(\cdot)$ admits the representation:

$$
k_{1}(z)=\sqrt{\frac{z-\frac{c^{2}}{2}}{z+\frac{c^{2}}{2}}}, \quad z \in \mathbb{C},
$$


where the branch of $\sqrt{\frac{z-\frac{c^{2}}{2}}{z+\frac{c^{2}}{2}}}$ is fixed by the condition $\sqrt{\frac{x-\frac{c^{2}}{2}}{x+\frac{c^{2}}{2}}}>0$ for $x>\frac{c^{2}}{2}$. We obtain the following:

$$
M^{D}(z)=\left(\begin{array}{cc}
m_{1}^{D}(z) & 0 \\
0 & m_{2}^{D}(z)
\end{array}\right), \quad z \in \mathbb{C}_{ \pm}
$$

where

$$
\begin{aligned}
& m_{1}^{D}(z):=c k_{1}(z) \tan (k(z) d) \\
& m_{2}^{D}(z):=-c k_{1}(z) \cot (k(z) d), \quad z \in \mathbb{C}_{ \pm},
\end{aligned}
$$

and $d:=\frac{b-a}{2}, \nu:=\frac{b+a}{2}$. The self-adjoint extension $D^{(1)}:=D^{*} \uparrow \operatorname{ker}\left(\Gamma_{0}^{D}\right)$ has the domain:

$$
\operatorname{dom}\left(D^{(1)}\right)=\left\{f \in W^{1,2}\left(\Delta, \mathbb{C}^{2}\right): f_{1}(a)=f_{1}(b)=0\right\}
$$

while the extension $D^{(2)}:=D^{*} \uparrow \operatorname{ker}\left(\Gamma_{1}^{D}\right)$ has the domain:

$$
\operatorname{dom}\left(D^{(2)}\right)=\left\{f \in W^{1,2}\left(\Delta, \mathbb{C}^{2}\right): f_{2}(a)=f_{2}(b)=0\right\} .
$$

In the following, we denote elements of $\mathfrak{L}$ by $\vec{f}$. In particular, we use the notation:

$$
\vec{f}=\left(\begin{array}{l}
\overrightarrow{f_{1}} \\
\overrightarrow{f_{2}}
\end{array}\right), \quad \vec{f}_{j} \in L^{2}\left(\Delta_{c}, \mathfrak{T}\right), \quad j=1,2
$$

Let us construct the boundary triplet $\Pi_{L}=\left\{\mathcal{H}^{L}, \Gamma_{0}^{L}, \widetilde{\Gamma}_{1}^{L}\right\}$ for $L^{*}$.

Operator $T$ gives us spectral decomposition $T=\oplus_{n=0}^{\infty} T_{n}$, where $T_{n}$ - bounded selfadjoint operator defined on $\mathfrak{T}_{n}, \mathfrak{T}=\oplus_{n=0}^{\infty} \mathfrak{T}_{n}$. Thus, operator $L$ admits the representation:

$$
L=\oplus_{n=0}^{\infty} L_{n}=\oplus_{n=0}^{\infty} D \otimes I_{\mathfrak{T}_{n}}+I_{\mathfrak{D}} \otimes T_{n} .
$$

For each $L_{n}$ boundary triplet, the Gamma field and the Weyl function can be obtained easily, as $T_{n}$ is bounded, see [6]. The problem is that the direct sum of boundary triplets, in general, is not a boundary triplet. The typical approach to such a problem is a regularization procedure, see [14]. However, in our case, the regularization is not necessary.

Theorem 3.1. The Weyl function $M^{L}(\cdot)$ is given by:

$$
M^{L}(z)=\left(\begin{array}{cc}
m_{1}^{D}(z-T) & 0 \\
0 & m_{2}^{D}(z-T)
\end{array}\right), \quad z \in \mathbb{C}_{ \pm} .
$$

Proof. We compute:

$$
C_{j}^{D}:=\sup _{\lambda \in \mathbb{R}}\left|m_{j}^{D}(i-\lambda)\right|<\infty, \quad \text { and } \quad \Lambda_{j}^{D}:=\sup _{\lambda \in \mathbb{R}} \frac{1}{\left|m_{j}^{D}(i-\lambda)\right|}<\infty
$$

$j=1,2$. This relies on the fact that $\sup _{\lambda \in \mathbb{R}}\left|k_{1}(i-\lambda)\right|<\infty$ and $\sup _{\lambda \in \mathbb{R}} \frac{1}{\left|k_{1}(i-\lambda)\right|}<\infty$.

Let us rewrite $L_{n}=D+n, n \in \mathbb{N}_{0}$ which is a closed symmetric operator defined on $\mathfrak{L}_{n}=\mathfrak{D} \otimes \mathfrak{T}_{n}$. Notice that $\mathfrak{L}=\oplus_{n \in \mathbb{Z}} \mathfrak{L}_{n}$ and $L=\oplus_{n \in \mathbb{Z}} L_{n}$. The triplet $\Pi_{L_{n}}=\Pi_{D} \otimes I_{\mathfrak{T}_{n}}=$ $\left\{\mathcal{H}^{L_{n}}, \Gamma_{0}^{L_{n}}, \Gamma_{1}^{L_{n}}\right\}$

$$
\mathcal{H}^{L_{n}}:=\mathcal{H}^{D} \otimes I_{\mathfrak{T}_{n}}, \quad \Gamma_{0}^{L_{n}}=\Gamma_{0}^{D} \otimes I_{\mathfrak{T}_{n}}, \quad \Gamma_{1}^{L_{n}}=\Gamma_{1}^{D} \otimes I_{\mathfrak{T}_{n}},
$$

is a boundary triplet for $L_{n}^{*}, n \in \mathbb{Z}$. The corresponding Weyl function $M^{L_{n}}(\cdot)$ is given by: 


$$
M^{L_{n}}(z)=\left(\begin{array}{cc}
m_{1}^{D}\left(z-T_{n}\right) & 0 \\
0 & m_{2}^{D}\left(z-T_{n}\right)
\end{array}\right), \quad n \in \mathbb{Z}, \quad z \in \mathbb{C}_{ \pm} .
$$

Notice that:

$$
C_{j n}^{D}:=\left\|m_{j}^{D}\left(i-T_{n}\right)\right\|=\sup _{\lambda \in \Delta_{n}}\left|m_{j}^{D}(i-\lambda)\right| \leq C_{j}^{D}<\infty, \quad j=1,2,
$$

which yields:

$$
\left\|M^{L_{n}}(i)\right\| \leq \max \left\{C_{1 n}^{D}, C_{2 n}^{D}\right\} \leq \max \left\{C_{1}^{D}, C_{2}^{D}\right\}<\infty .
$$

Similarly, we verify:

$$
\left\|\left(M^{L_{n}}(i)\right)^{-1}\right\| \leq \max \left\{\Lambda_{1 n}^{D}, \Lambda_{2 n}^{D}\right\} \leq \max \left\{\Lambda_{1}^{D}, \Lambda_{2}^{D}\right\}<\infty,
$$

where:

$$
\Lambda_{j n}^{D}:=\left\|\left(m_{j}^{D}\left(i-T_{n}\right)\right)^{-1}\right\|=\sup _{\lambda \in \Delta_{n}} \frac{1}{\left|m_{j}^{D}(i-\lambda)\right|}<\Lambda_{j}^{D}<\infty,
$$

$j=1,2$. By Theorem 2.5, we see that $\Pi_{L}^{\prime}=\oplus_{n \in \mathbb{Z}} \Pi_{L_{n}}$ is a boundary triplet for $L$. In particular, the Weyl function $M^{L}(\cdot)$ is computed by:

$$
M^{L}(z)=\left(\begin{array}{cc}
m_{1}^{D}(z-T) & 0 \\
0 & m_{2}^{D}(z-T)
\end{array}\right), \quad z \in \mathbb{C}_{ \pm} .
$$

The considerations remain true if we use the boundary triplet of [7, Section 3.1].

\section{Acknowledgments}

This work was partially financially supported by the Government of the Russian Federation (grant 074-U01), by Ministry of Science and Education of the Russian Federation (GOSZADANIE 2014/190, Projects No 14.Z50.31.0031 and No. 1.754.2014/K), by grants MK-5001.2015.1 and MK-5161.2016.1 of the President of the Russian Federation, by grant 16-11-10330 of Russian Science Foundation. The first author acknowledge hospitality and financial support of WIAS Berlin. The second author thanks the European Research Council and the DFG supporting the preparation of the paper via ERC-2010-AdG no. 267802 (Analysis of Multiscale Systems Driven by Functionals) and Grant NE 1439/3-1 (Development of Methods in the Theory of Self-adjoint Extensions), respectively.

\section{References}

[1] Pan L., Fu X., Zhou G. Electron dwell time through a quantum wire under a electromagnetic field irradiation. Phys. Lett. A, 2007, 368(1-2), P. 97-100.

[2] Popov I.Y. Operator extensions theory model for electromagnetic field-electron interaction. J. Math. Phys., 2012, 53, P. 063505.

[3] Neidhardt H., Wilhelm L., Zagrebnov V.A. A new model for quantum dot light emitting-absorbing devices. J. Math. Phys., Anal., Geom., 2014, 10(3), P. 350-385.

[4] Neidhardt H., Wilhelm L., Zagrebnov V.A. A new model for quantum dot light emitting-absorbing devices: proofs and supplements. Nanosystems: Phys. Chem. Math., 2015, 6(1), P. 6-45.

[5] Albeverio S., Brasche J.F., Malamud M.M., Neidhardt H. Inverse spectral theory for symmetric operators with several gaps: scalar-type Weyl functions. J. Funct. Anal., 2005, 228(1), P. 144-188,

[6] Boitsev A.A. ,Neidhardt H., Popov I.Y. Weyl function for sum of operators tensor products. Nanosystems: Phys. Chem. Math., 2013, 4(6), P. 747-757. 
[7] Carlone R., Malamud M., Posilicano A. On the spectral theory of Gesztesy-Šeba realizations of 1-D Dirac operators with point interactions on a discrete set. J. Differential Equations, 2013, 254(9), P. 3835-3902.

[8] Derkach V.A., Malamud M.M. On the Weyl function and Hermite operators with lacunae. Dokl. Akad. Nauk SSSR, 1987, 293(5), P. 1041-1046.

[9] Derkach V.A., Malamud M.M. Generalized resolvents and the boundary value problems for Hermitian operators with gaps. J. Funct. Anal., 1991, 95(1), P. 1-95.

[10] Derkach V.A., Malamud M.M. The extension theory of Hermitian operators and the moment problem. $J$. Math. Sci., 1995, 73(2), P. 141-242.

[11] Gorbachuk V.I., Gorbachuk M.L. Boundary value problems for operator differential equations, volume 48 of Mathematics and its Applications (Soviet Series). Kluwer Academic Publishers Group, Dordrecht, 1991.

[12] Kočubě̀A. N. Extensions of symmetric operators and of symmetric binary relations. Mat. Zametki, 1975, 17, P. 41-48.

[13] Malamud M.M. Some classes of extensions of a Hermitian operator with lacunae. Ukraïn. Mat. Zh., 1992, 44(2), P. 215-233.

[14] Malamud M.M., Neidhardt H. Sturm-Liouville boundary value problems with operator potentials and unitary equivalence. J. Differential Equations, 2012, 252(11), P. 5875-5922.

[15] Schmüdgen K. Unbounded self-adjoint operators on Hilbert space, 2012, volume 265 of Graduate Texts in Mathematics. Springer, Dordrecht. 